\title{
Review of laser speckle contrast techniques for visualizing tissue perfusion
}

\author{
Matthijs Draijer • Erwin Hondebrink • \\ Ton van Leeuwen · Wiendelt Steenbergen
}

Received: 9 October 2008 / Accepted: 14 October 2008 / Published online: 3 December 2008

(C) The Author(s) 2008. This article is published with open access at Springerlink.com

\begin{abstract}
When a diffuse object is illuminated with coherent laser light, the backscattered light will form an interference pattern on the detector. This pattern of bright and dark areas is called a speckle pattern. When there is movement in the object, the speckle pattern will change over time. Laser speckle contrast techniques use this change in speckle pattern to visualize tissue perfusion. We present and review the contribution of laser speckle contrast techniques to the field of perfusion visualization and discuss the development of the techniques.
\end{abstract}

Keywords LASCA · LSI - LSFG • Speckle contrast • Perfusion

\section{Introduction}

Imaging blood flow in the tissue is of major importance in the clinical environment [1-10]. Over recent decades, several techniques have been developed for imaging tissue perfusion. Most of these techniques [10-13]

M. Draijer $(\bowtie)$ · E. Hondebrink · T. van Leeuwen .

W. Steenbergen

Biophysical Engineering Group Institute for Biomedical

Technology University of Twente, Enschede,

The Netherlands

e-mail: m.j.draijer@utwente.nl

T. van Leeuwen

Biomedical Engineering \& Physics, Academic Medical

Center University of Amsterdam, Amsterdam,

The Netherlands exploit the interference pattern generated from diffusely backscattered light from the skin [14]. Currently, laser speckle contrast techniques are gaining interest $[15,16]$. Laser speckle contrast techniques are based on the spatial and temporal statistics of the speckle pattern. The motion of particles in the illuminated medium causes fluctuations in the speckle pattern on the detector. These intensity fluctuations blur the image and reduce the contrast to an extent that is related to the speed of the illuminated objects, such as moving red blood cells. In this paper, we will present the principles and various implementations of the speckle contrast method, review the contribution of laser speckle contrast techniques to the field of perfusion imaging, and describe their technical development.

\section{Speckle contrast}

What are speckles?

When an optically rough object is illuminated with coherent laser light and the diffusely backscattered light is collected on a screen, the backscattered light will create an interference pattern on this screen. This interference pattern consists of bright and dark areas; the so-called speckles. If the object does not move and the laser is stable, the interference pattern does not change over time, and the pattern is called a static speckle pattern. If the object moves or particles move within the medium, the interference pattern will change in time and the pattern is called dynamic. This dynamic behavior is mainly caused by the Doppler shifts of the light that interacts with the moving particles. Figure 1a shows a 


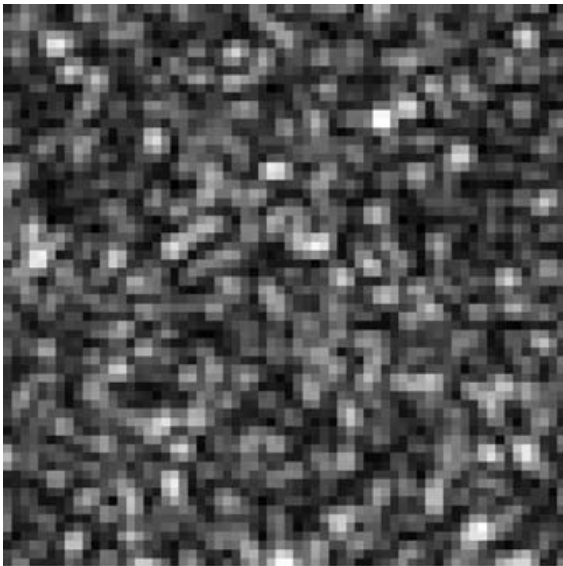

(a)

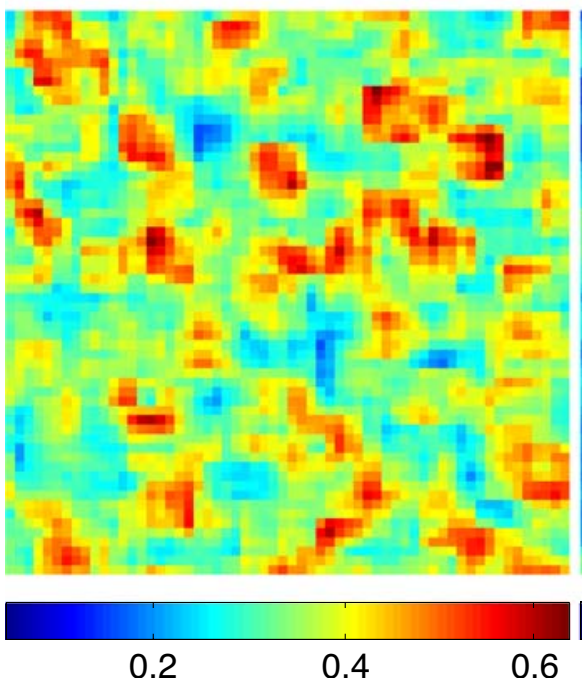

(d)

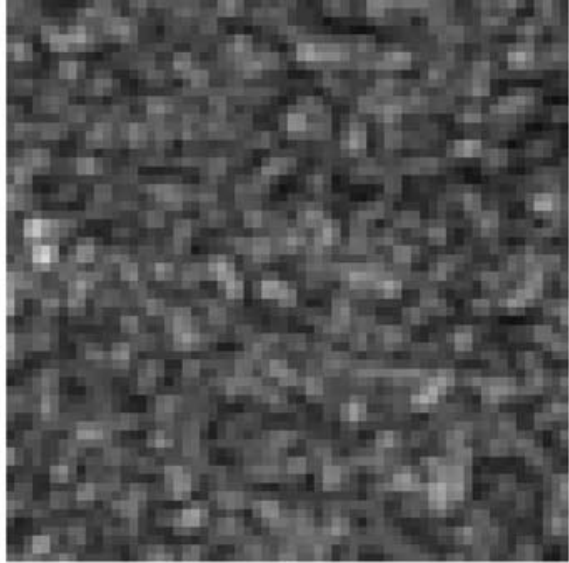

(b)

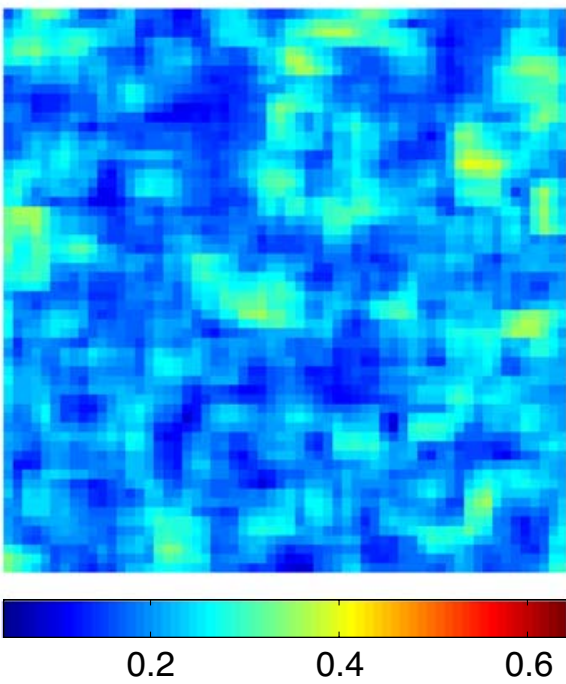

(e)

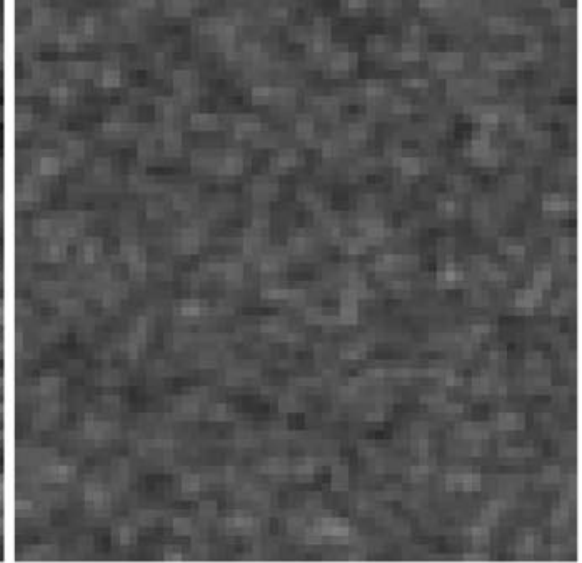

(c)

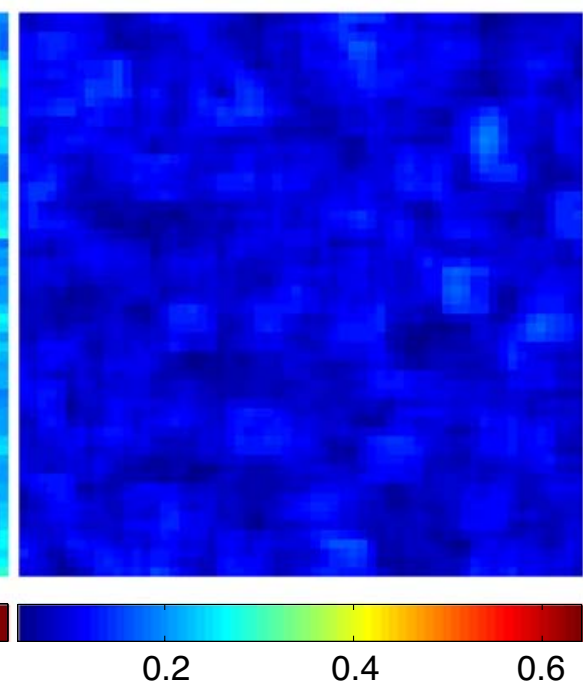

(f)

Fig. 1 a, b and $\mathbf{c}$ Simulated blurred speckle patterns with an exposure time of 1, 5, and 25 ms respectively, d, e, and $\mathbf{f}$ conjugated contrast images, with contrast values calculated for $5 \times 5$ pixels. The contrast value is shown in the colorbar

simulated speckle pattern formed when the diffusely backscattered light is emitted from a circular area [17].

What is speckle contrast?

Speckle flow techniques are based on the changes over time of the dynamic speckle pattern generated by motion in the sample. In these techniques, this changing speckle pattern is recorded with a camera that has an integration time in the order of the speckle decorrelationtime (i.e., in the millisecond range). Due to the long integration time compared to the typical decorrelationtime of the speckle pattern, the speckle pattern will be blurred in the recorded image. The level of blurring is quantified by the speckle contrast. The speckle contrast $C$ is usually defined as the ratio of the standard deviation $\sigma$ of the intensity $I$ to the mean intensity $\langle I\rangle$ of the speckle pattern:

$C \equiv \frac{\sigma}{\langle I\rangle}=\frac{\sqrt{\left\langle I^{2}\right\rangle-\langle I\rangle^{2}}}{\langle I\rangle}$

If there is no or little movement in the object, there will be no or only a little blurring. Goodman and Parry [18] showed that for a static speckle pattern, under ideal conditions (i.e., perfectly monochromatic and polarized waves and absence of noise) the standard deviation $\sigma$ equals the mean intensity $\langle I\rangle$ and the speckle contrast is equal to unity, which is the maximum value for the contrast. Such a speckle pattern is termed "fully developed". When there is movement in the object, the speckle pattern will be blurred and the standard deviation of the intensity will be small compared to 
the unchanged mean intensity, resulting in a reduced speckle contrast. Figure 1 shows three simulated speckle images with different exposure times and their corresponding contrast maps. The latter are obtained by calculating the contrast over an area of $5 \times 5$ pixels. The speckle images are simulated by making use of the concept of a copula [17]: a circular region in a square matrix is filled with complex numbers of unity amplitude and uniformly distributed phases. After Fourier transforming the matrix, and multiplying it point-bypoint by its complex conjugate, an artificial speckle pattern is generated. By shifting the circular region with complex numbers one column each time and recalculating the speckle pattern, a dynamic speckle pattern can be generated. The speckle pattern is decorrelated if all the complex numbers in the circular region are changed (i.e., after the same amount of steps as the diameter of the circular region). So the diameter of the circular region can be related to the speckle decorrelation time, in this way different exposure times can be simulated.

\section{Theories relating speckle contrast to particle speed}

A dynamic speckle pattern can be described in terms of a power spectrum of the intensity fluctuations. In the time domain, an analogous description is by the autocorrelation function of the intensity fluctuations. An important feature of such a temporal correlation function is the decorrelation time. Under the assumption of a random Gaussian or Lorentzian velocity distribution with a mean around zero, the decorrelation time $\tau_{c}$ can be linked to the decorrelation velocity $v_{c}[19-21]$ by:

$v_{c}=\frac{\lambda}{2 \pi \tau_{c}}$

with $\lambda$ the laser wavelength. Using laser light in the visible range, this relation reduces to $v_{c} \approx 0.1 / \tau_{c} \mu \mathrm{m} / \mathrm{s}$. Bonner and Nossal [22] took more factors such as particle size into account and reduced the relation to $v_{c} \approx$ $3.5 / \tau_{c} \mu \mathrm{m} / \mathrm{s}$. So the decorrelation velocity predicted by Bonner and Nossal differs by one and a half orders of magnitude from the values predicted by Briers and Webster. Which of these relations best predicts the decorrelation velocity is yet unknown.

For laser speckle contrast techniques, the particle velocity and/or the speckle decorrelation time need to be related to the speckle contrast. Ramirez-San-Juan et al. [23] investigated the influence of a Gaussian or Lorentzian velocity distribution on the contrast level. Under the assumption of a Lorentzian [23, 24] velocity distribution, the relation between the correlation time $\tau_{c}$, the exposure time $T$ and the contrast is given by:

$\frac{\sigma}{\langle I\rangle}=\sqrt{\frac{\tau_{c}}{2 T}\left(1-\exp \left(-\frac{2 T}{\tau_{c}}\right)\right)}$

So a small value of the contrast corresponds with a small $\tau_{c}$ (i.e., fast-moving speckles) and a contrast close to unity corresponds to a large $\tau_{c}$ (i.e., stationary speckle pattern). For a Gaussian velocity distribution $[19,23,25]$, the relation is given by:

$\frac{\sigma}{\langle I\rangle}=\sqrt{\frac{\sqrt{\pi}}{2} \frac{\tau_{c}}{T} \operatorname{erf}\left(\frac{T}{\tau_{c}}\right)}$

The integral over time of the normalized autocorrelation function of a velocity distribution should equal the correlation time $\tau_{c}$. However, for Eq. 4 this is not the case, so Ramirez-San-Juan et al. [25] proposed to use an alternate expression for the Gaussian velocity distribution :

$$
\frac{\sigma}{\langle I\rangle}=\sqrt{\frac{1}{2} \frac{\tau_{c}}{T} \operatorname{erf}\left(\frac{\sqrt{\pi} T}{\tau_{c}}\right)}
$$

In Fig. 2, $C$ is plotted as a function of $T / \tau_{c}$ under the assumption of a Lorentzian and the two Gaussian velocity distributions. There is a clear difference visible between the contrast values based on a Lorentzian or Gaussian velocity distribution for a given $T / \tau_{c}$. For high contrast levels, correlation times may vary up to one order of magnitude. However, for the alternate Gaussian velocity distribution, there is a good agreement between the Lorentzian and Gaussian velocity distribution for $C$-values below 0.5. Ramirez-San-Juan

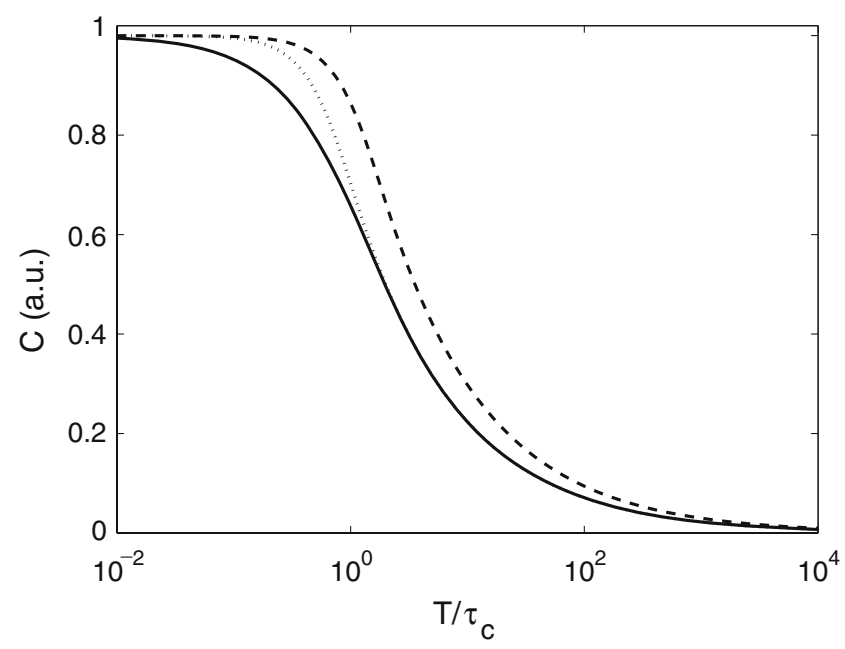

Fig. $2 \mathrm{C}$ as a function of $T / \tau_{c}$ for a Lorentzian velocity distribution (solid), Gaussian velocity distribution (dashed) and alternate Gaussian velocity distribution (dotted) 
et al. [23] showed furthermore that for the flow rates used in their experiments, the Gaussian based approach is superior to the normally used Lorentzian approach in speckle contrast techniques. The relation between $\tau_{c}$ and $v_{c}$ (e.g., Eq. 2) is essential to link the measured contrast values via speckle decorrelation time to the decorrelation velocity.

Recently, Duncan et al. [26] stated that the Lorentzian velocity distribution model is only applicable for Brownian motion whereas an inhomogeneous (Gaussian) distribution is valid for ordered motion. They claimed that the proper model for the combined effect (i.e., Brownian motion and ordered motion) is a Voigt velocity distribution, which is the result of a convolution of a Lorentzian and Gaussian velocity distribution.

\section{Speckle contrast flow measurement techniques}

Several researchers used the principle of speckle contrast to develop techniques for measuring skin perfusion. In this paper, several of these techniques will be discussed. Table 1 summarizes the various techniques.

Double- and single-exposure speckle photography

Archbold and Ennos [27] invented double-exposure speckle photography $[28,29]$, a technique which was the forerunner of LASCA (see Section Laser speckle contrast analysis (LASCA)). Strictly speaking, doublespeckle photography is not a speckle contrast technique since each of the two exposures is a snapshot rather than a blurred image. Double-exposure speckle photography is based on the principle that a photographic record of two identical and mutually displaced speckle structures gives rise to parallel straight fringes in the Fourier plane. The spacing and orientation of these fringes is related to the displacement and direction between both photographs. This makes the technique only applicable for solid bodies or fluids with a stationary flow pattern [27]. Iwai and Shigeta [30] developed a digital version of double-exposure speckle photography. To obtain a velocity map, the whole image should be divided into small regions for analysis over which the velocity can be assumed to be spatially constant. The analysis of these fringe-patterns is complicated compared to analysis performed in speckle contrast techniques, which is a disadvantage.

The first real speckle contrast technique, using a long exposure time, was single-speckle photography [31]. Single-exposure speckle photography [24] was a laborious process (i.e., making and developing a photograph and analysis of the negative film).

Laser speckle contrast analysis (LASCA)

Briers and Webster [19, 32, 33] developed a digital version of single speckle photography using a

Table 1 Methods of measuring tissue blood flow with laser speckle contrast techniques

\begin{tabular}{|c|c|c|c|}
\hline Technique & Abbrev. & Domain & Principle \\
\hline $\begin{array}{l}\text { Laser speckle contrast } \\
\text { analysis }[19,51]\end{array}$ & LASCA & Spatial & Contrast is determined in 1 image over $5 \times 5$ or $7 \times 7$ pixels. \\
\hline Laser speckle imaging [42] & LSI & Temporal & Contrast is determined in 1 pixel over 25 or 49 images. \\
\hline $\begin{array}{l}\text { Double exposure speckle } \\
\text { photography }[28,30]\end{array}$ & DESP & Spatial & $\begin{array}{l}\text { A sequence of two rapid speckle recordings is taken in } 1 \text { image. } \\
\text { The resulting fringes contain information about the movement. }\end{array}$ \\
\hline $\begin{array}{l}\text { Single exposure speckle } \\
\text { photography }[19,24]\end{array}$ & SESP & Spatial & Forerunner of LASCA, based on the same principle. \\
\hline $\begin{array}{l}\text { Laser speckle temporal contrast } \\
\text { analysis [45] }\end{array}$ & LSTCA & Temporal & Contrast is determined in one pixel over a sequence of images. \\
\hline $\begin{array}{l}\text { Laser speckle perfusion } \\
\text { imaging }[52,53]\end{array}$ & LSPI & Spatial \& temporal & Combination of LASCA and LSI. \\
\hline Laser speckle flowgraphy [54] & LSFG & Spatial \& temporal & $\begin{array}{l}\text { The contrast is determined based on an area of } 3 \times 3 \text { pixels, } \\
\text { in } 3 \text { speckle images. }\end{array}$ \\
\hline $\begin{array}{l}\text { Spatial derived contrast } \\
\text { with averaging [55] }\end{array}$ & SDCav & Spatial & $\begin{array}{l}\text { Contrast is determined based on averaging a sequence } \\
\text { of LASCA-images. }\end{array}$ \\
\hline $\begin{array}{l}\text { Temporal laser speckle contrast } \\
\text { analysis [56] }\end{array}$ & tLASCA & Spatial \& temporal & $\begin{array}{l}\text { Contrast is determined based on averaging a sequence } \\
\text { of LSI-images. }\end{array}$ \\
\hline $\begin{array}{l}\text { Spatial laser speckle contrast } \\
\text { analysis [56] }\end{array}$ & sLASCA & Spatial & $\begin{array}{l}\text { Contrast is determined based on averaging a sequence } \\
\text { of LASCA-images. }\end{array}$ \\
\hline $\begin{array}{l}\text { Multi-exposure speckle } \\
\text { imaging [50] }\end{array}$ & MESI & Spatial & $\begin{array}{l}\text { Contrast is determined in } 1 \text { image over } 7 \times 7 \text { pixels. } \\
\text { Exposure time is kept constant and } T \text { is controlled } \\
\text { by laser pulse duration. }\end{array}$ \\
\hline
\end{tabular}




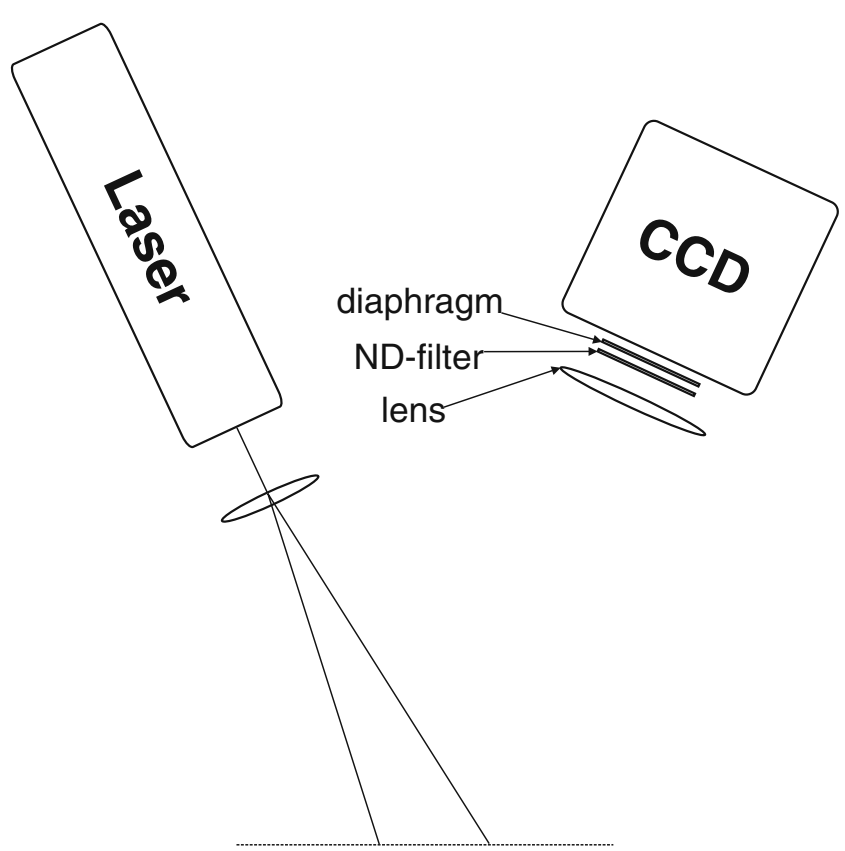

(a)

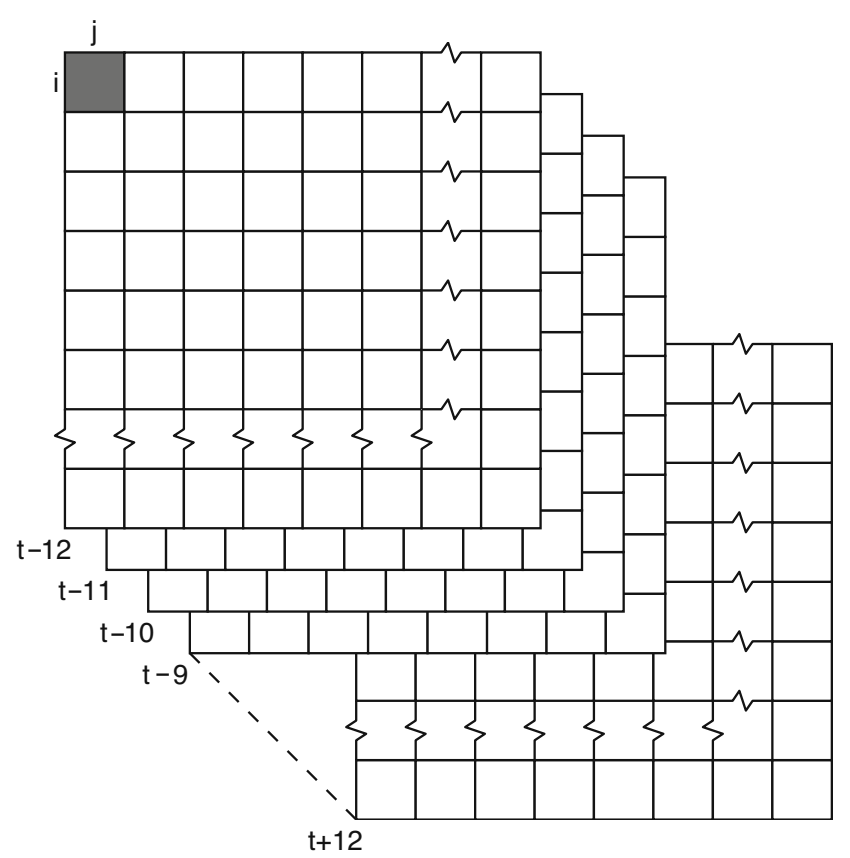

(c)

Fig. 3 a LASCA-setup with essential features. b Schematic overview of the way the contrast is calculated in LASCA. With the squares being pixels of a recorded image, the contrast in pixel (i,j) (dark grey) is determined by calculating the ratio of the standard deviation of the pixels in the pale grey $n \times n$ pixel area to the mean value of the pixels in this area. c Schematic overview of the way the contrast is calculated in LSI. In pixel $(i, j)$

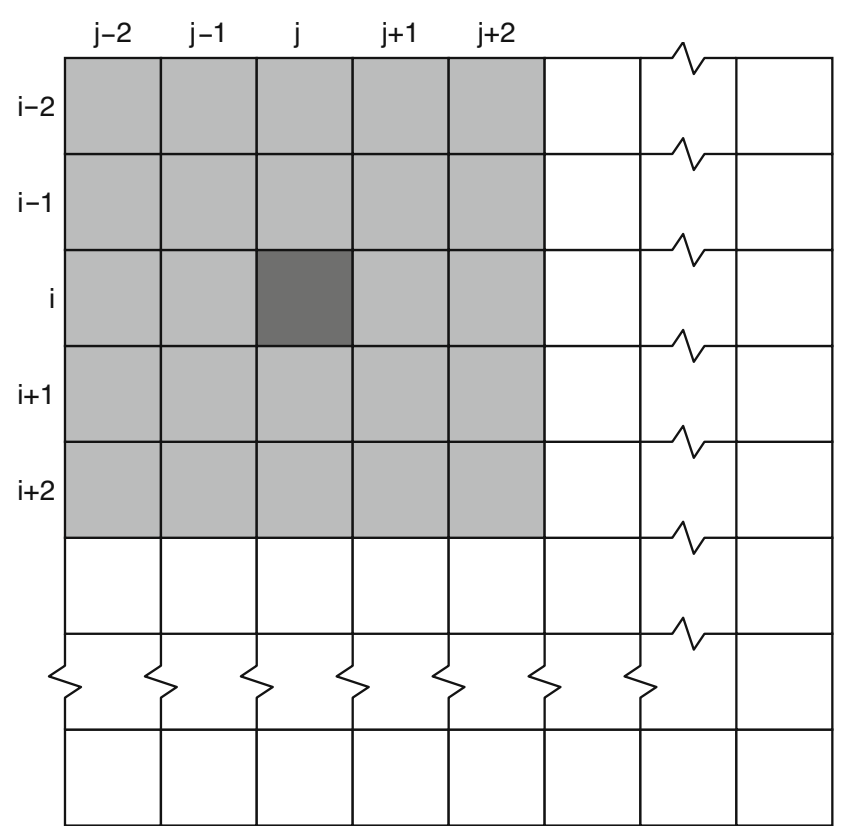

(b)

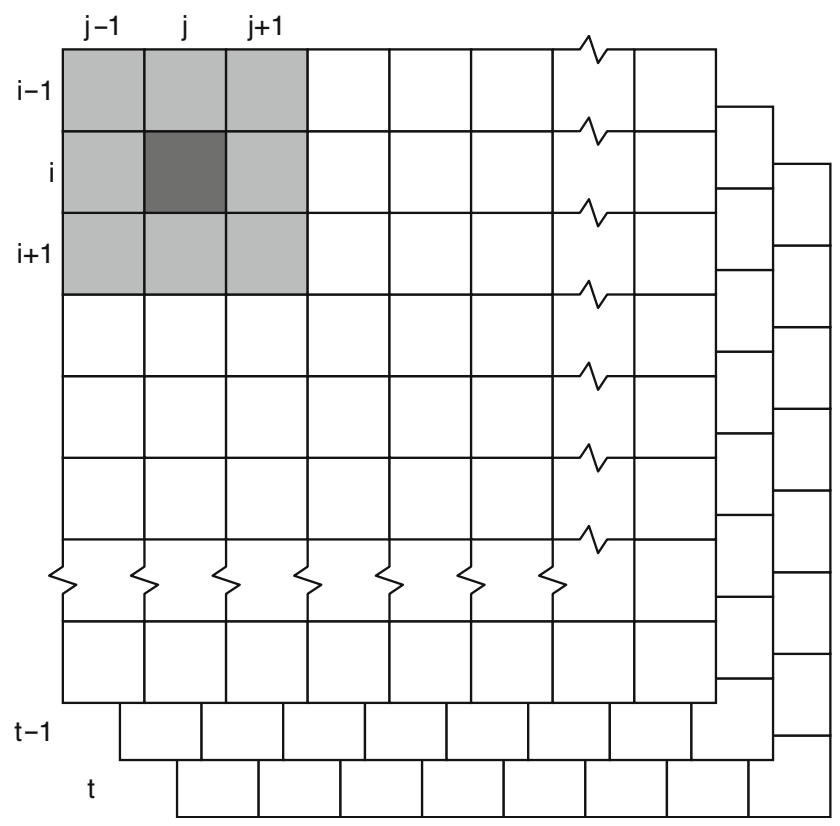

$t+1$

(d)

the contrast is calculated as the ratio of the standard deviation of the intensity at this pixel at different times, and the mean intensity for this pixel and $\mathbf{d}$ schematic overview of the way the contrast is calculated in LSFG. The mean blur rate (MBR) is determined by calculating the ratio of the mean value of the pixels in the pale gray area to mean difference of the central point (dark gray) and the pixels in the pale gray area 
monochrome CCD and frame grabber linked to a computer. The digital photograph is processed by the computer and the local contrast is computed in a block of $n \times n$ pixels. This digital version was the first setup which uses Laser Speckle Contrast Analysis (LASCA) as we know it nowadays. Figure 3 a shows a schematic overview of a LASCA setup with an expanded laser beam, an imaging system comprising focussing optics, a variable diaphragm and a digital camera as essential features. Figure $3 b$ shows a schematic overview of the way the contrast is calculated in LASCA. The contrast is calculated by:

$$
C_{i, j}=\frac{\sqrt{\frac{1}{(n+1)^{2}} \sum_{x=i-\frac{n}{2}}^{i+\frac{n}{2}} \sum_{y=j-\frac{n}{2}}^{j+\frac{n}{2}} I_{x, y}^{2}-\left(\frac{1}{(n+1)^{2}} \sum_{x^{\prime}=i-\frac{n}{2}}^{i+\frac{n}{2}} \sum_{y^{\prime}=j-\frac{n}{2}}^{j+\frac{n}{2}} I_{x^{\prime}, y^{\prime}}\right)^{2}}}{\frac{1}{(n+1)^{2}} \sum_{x=i-\frac{n}{2}}^{i+\frac{n}{2}} \sum_{y=j-\frac{n}{2}}^{j+\frac{n}{2}} I_{x, y}}
$$

with $I_{i, j}$ the intensity of pixel $i, j$ and $n+1$ the size of the square over which the contrast is calculated. Experiments showed [34, 35] that in LASCA it was not possible to obtain the full contrast range from 0 to 1.0. Richards and Briers $[35,36]$ suggested this was due to an offset in the pixel values termed pedestal and introduced by the CCD camera. Manually removing this offset resulted in an increase in contrast from 0.41 to 0.95 for a static speckle pattern. The next major improvement to the LASCA setup was in the image processing. Changing from software written in $\mathrm{C}^{++}$ operating under DOS to Windows in combination with improved code and a 166-MHz Pentium processor, reduced the processing time for a full frame from approximately 4 min down to approximately $1 \mathrm{~s}[35,37]$. Besides improving the computer hardware and software, the development of the LASCA technique continued. An improved version was described by Richards and Briers who implemented a camera with a variable exposure time and ran trials with lasers in the green wavelength range instead of in the red $[21,35,36]$. Several researchers used a slightly different LASCA setup. For example, in one setup, the backscattered light was collected on the camera without making use of a lens but by making use of a singlemode fiber [38] or adjustable iris and camera in the diffraction plane [39]. Furthermore, a polarizer was positioned in between sample and camera to select the linearly polarized light [40] to increase the contrast of the grabbed speckle pattern $[21,35,36,38]$.

LASCA is fast and inexpensive, but there are technical details which should be taken into account for proper measurements. To adjust the "sensitivity" of the LASCA setup to a certain velocity, the integration time can be adjusted. As the integration time changes, the noise in the measurement also changes. Yuan et al. [41] identified a relation linking sensitivity, noise, and camera exposure time. They found that with an increasing exposure time up to $2 \mathrm{~ms}$, the sensitivity to relative speckle changes increased. However, the noise in the speckle contrast also increases with increasing exposure time. The optimal contrast-to-noise ratio was found to be at $5 \mathrm{~ms}$, so Yuan et al. suggested that $\sim 5 \mathrm{~ms}$ is an optimal exposure time for LASCA measurements in the brains of rodents.

To obtain good statistics, the speckle size should be carefully controlled. When speckle size and pixel size are of the same order, the error in calculated contrast is minimized [35, 41]. For image speckle (i.e., image the speckle pattern with a lens in front of for example a camera), the speckle size is dependent on the laser wavelength $(\lambda)$, the f-number of the lens system $\left(f_{\#}\right)$ and the magnification $(M)$, as expressed by:

$d=2.44 \lambda f_{\#} M$

So by controlling the f-number of the lens system (i.e., adjusting the iris of the lens system) the optimal speckle size can be chosen. However, this removes the facility to control the amount of light falling on the camera because Yuan et al. [41] showed that a fixed integration time of $\sim 5 \mathrm{~ms}$ gives the best contrast-to-noise ratio. So neutral density filters should be used to adjust the amount of light falling on the camera [35].

With "classical" LASCA, all depth information about perfusion is lost, so Zimnyakov and Misnin [39] modified the setup by making use of a localized moving light source in combination with spatial filtering to reveal depth-resolved information about the micro circulation. When a dynamic layer below a static layer is imaged, the resulting speckle pattern will be composed of a stationary speckle pattern in the inner zone of the CCD camera and a dynamic speckle pattern in the outer zone. So by placing filtering diaphragms on the sample, depth information can be obtained. As a consequence of the stationary speckle pattern, the contrast will not drop to 0 for long integration times. To quantify that Zimnyakov and Misnin introduced the term residual contrast.

\section{Laser speckle imaging (LSI)}

Due to the fact that the contrast is analyzed for a group of pixels in one image, LASCA has the disadvantage 
of a lack of spatial resolution. So Cheng et al. [42] developed Laser Speckle Imaging (LSI) to compensate for this disadvantage. LSI is the temporal equivalent of LASCA; the contrast is calculated based on one pixel in a time sequence, rather than based on multiple pixels in one image, as is schematically shown in Fig. 3(c). In LSI, the contrast is calculated by:

$$
C_{i, j}=\frac{\sigma}{\langle I\rangle}=\frac{\sqrt{\frac{1}{n+1} \sum_{l=t-n / 2}^{t+n / 2} I_{i, j, l}^{2}-\left(\frac{1}{n+1} \sum_{l^{\prime}=t-n / 2}^{t+n / 2} I_{i, j, l^{\prime}}\right)^{2}}}{\frac{1}{n+1} \sum_{l=t-n / 2}^{t+n / 2} I_{i, j, l}}
$$

where $I_{x, y, t}$ is the intensity of pixel $(i, j)$ at time $t$ and $n+1$ the number of speckle images over which the contrast is calculated. Note that no flow (i.e., no dynamic speckle pattern) and very high flow (i.e., complete blurred dynamic speckle pattern) both give a contrast equal to 0 . This makes LSI unsuitable for sample with regions where no flow is present. Cheng et al. [42] showed that calculating the contrast with LSI gives as expected a five times higher spatial resolution compared to LASCA. They only assumed a linear relationship between the measured flow rate (i.e., $1 / \tau_{c}$ ) and actual flow rate values, whereas Choi et al. [43] showed that there is a linear relationship between these parameters, the range over which this is valid depends on the integration time of the camera (e.g., 0 to $20 \mathrm{~mm} / \mathrm{s}$ for $\mathrm{T}=1 \mathrm{~ms}$ and 0 to $5 \mathrm{~mm} / \mathrm{s}$ for $\mathrm{T}=10 \mathrm{~ms}$ ), as already was suggested by Yuan et al. [41].

Nothdurft and Yao [44] showed that by adjusting the capture parameters (e.g., exposure time, incident power, and time interval between subsequent capture), LSI is able to reveal structures that are hidden under the surface. Surface and subsurface inhomogeneities depend differently on these capture parameters, so by tuning the capture parameters, the image contrast values of the surface and subsurface targets can be changed. When the contrast of the surface inhomogeneity is within the noise level of the background image, the surface effect is essentially removed from the image. They did not test LSI on tissue perfusion; that was done by Li et al. [45] who named the technique differently, Laser Speckle Temporal Contrast Analysis (LSTCA), but it is based on the same principle as LSI. They presented images of the cerebral blood flow of a rat through the intact skull by making use of temporal averaging of the speckle pattern. They used an exposure time of $5 \mathrm{~ms}$, which is of the same order as that suggested by Yuan et al. [41] and an interval time of 25 ms, resulting in a real-time video frame rate of $33 \mathrm{~Hz}$. They furthermore showed that LSTCA significantly improves the visualization of the blood vessels with respect to LASCA due to the fact that the speckle pattern on the detector is built up of a stationary and a dynamic part. They stated that the stationary part produced by the skull is mainly dependent on local properties of the skull and is therefore temporally homogeneous. So the contrast value in the LSTCA process is not influenced by the stationary part, whereas in the LASCA process, the stationary part will influence the contrast value and lower the SNR.

Völker et al. [46] modified LSI by positioning a rotating diffuser, which can be controlled by a motor, to illuminate the sample with a random speckle pattern. In this way, they could suppress the noise in LSI. If the diffuser rotates slowly (e.g., one rotation per hour), temporal fluctuations will occur at time scale $\tau_{0}$. However, if the exposure time $T$ of the camera is chosen to be smaller than $\tau_{0}$, subsequent speckle images will be statistically independent and analyzing a large number of images results in the perfect averaging of the contrast without loss of spatial resolution. They showed that the noise level scales with $N^{-0.5}$, with $N$ being the number of independent speckle images.

Bandyopadhyay et al. [47] and Zakharov et al. [48] pointed out recently that the commonly used LSI equation (i.e., Eq. 3) involves an approximation (i.e., $\tau_{c} \ll$ $T$ for Lorentzian velocity distribution) that could result in incorrect data analysis. Cheng and Duong [49] investigated the contribution of such approximation and its impact on LSI data analysis. They showed that the approximation is valid for calculating blood flow changes rather than absolute values for $\tau_{c} \ll T$.

Furthermore, they introduced a time-efficient LSI analysis method by making use of the asymptotic approximation of the commonly used LSI equation (i.e., Eq. 3) instead of using the Newtonian iterative method to solve that equation. Based on these findings, Parthasarathy et al. [50] presented a new multiexposure speckle imaging (MESI) instrument based on their robust speckle model that has potential to obtain quantitative baseline flow measures and overcomes their criticism of LASCA and LSI (e.g., lack of quantitative accuracy and the inability to predict flows in the presence of static scatterers such as an intact or thinned skull). To keep the noise contribution of the camera (e.g., readout noise, thermal noise) constant while changing the integration time, they used a fixed exposure time for the camera and gated a laser diode during each exposure to effectively vary the speckle exposure duration $T$. 
Other techniques

LASCA has the disadvantage of a lack of spatial resolution whereas LSI has the disadvantage of a lack of temporal resolution. Therefore, several researchers [52-56] have developed techniques which are combinations of LASCA and LSI. Forrester et al. [52, 53] developed Laser Speckle Perfusion Imaging (LSPI), Tan et al. [55] developed LASCA using Spatially Derived Contrast with Averaging (SDCav), Konishi et al. [54] developed Laser Speckle Flowgraphy (LSFG) and Le et al. [56] introduced $t L A S C A$ and $s L A S C A$ as temporal and spatial equivalents of LASCA.

In LSPI, the mean value of the speckle intensity, which is called speckle reduction by Forrester et al. [52, 53], can be determined by spatial averaging (good temporal resolution), temporal averaging (good spatial resolution) or a combination of both (acceptable temporal and spatial resolutions). The nonfluctuating component of the measured intensity is quantified by the speckle reduction:

$$
\begin{aligned}
& I_{R E F}(i, j) \\
& \quad=\frac{1}{N_{M A X}} \sum_{N=1}^{N_{M A X}}\left[\frac{1}{2 m+1} \sum_{i-m}^{i+m}\left(\frac{1}{2 n+1} \sum_{j-n}^{j+n} I_{S P, N}(i, j)\right)\right]
\end{aligned}
$$

where $I_{S P, N}$ is the intensity of pixel $(i, j)$ in the $N^{t h}$ frame in a sequence of $N_{M A X}$ frames and $m$ an $n$ are the boundaries for the chosen region around pixel $(i, j)$. To quantify the fluctuating component, the sum of the difference between the speckle reduction and the speckle intensity is taken and normalized with the speckle reduction:

$$
\begin{array}{r}
I_{S D, \text { norm }}(i, j) \\
=\frac{\sum_{N=1}^{N_{M A X}}\left[\sum_{i-m}^{i+m}\left(\sum_{j-n}^{j+n}\left|I_{S P, N}(i, j)-I_{R E F}(i, j)\right|\right)\right]}{I_{R E F}(i, j)}
\end{array}
$$

which is different from the formal definition of contrast as given in Eq. 1, where the numerator is based on the standard deviation of the fluctuation instead of the mean absolute difference of the fluctuations.

To determine the perfusion, the inverse relation of the normalized sum is taken. For obtaining high spatial resolution images, Forrester et al. [53] used a frame rate of just over $6 \mathrm{~Hz}$, while with spatial averaging they obtained a semi-real-time imaging speed with a frequency of $15 \mathrm{~Hz}$. The method of calculating the flow in LSFG, or mean blur rate (MBR) as Konishi et al. [54] termed it, is comparable to the combination of spatial and temporal averaging introduced by Forrester et al. [53]. In LSFG a $3 \times 3 \times 3$ pixel matrix is taken and the MBR is defined as the mean intensity across these 26 pixels (the central point is not taken into account) divided by the mean difference of the central point and the 26 pixels. This is schematically shown in Fig. 3d. When using a CCD camera in LSFG, the interlace scanning mode of the camera requires compensation for the fact the odd lines are captured at different time to the even lines, so Konishi et al. [54] adjusted the definition of the MBR in LSFG to:

$$
M B R_{n, m, t}=\frac{2\left\langle I_{n, m, t}\right\rangle^{2}}{\left\langle I_{n, m, t}^{2}\right\rangle-\left\langle I_{n, m, t}\right\rangle^{2}}
$$

where the factor 2 in the numerator is related to the number of uncorrelated intensity data taken for the averaging (i.e., even and odd lines).

Tan et al. [55] modified the "classical" LASCA to SDCav by introducing averaging over multiple contrast maps, resulting in a decrease in root mean square (RMS) of the value of $1 / \tau_{c}$ with an increasing number of averages. A few years later, this technique of averaging over multiple contrast maps was presented by Le et al. [56] under the name sLASCA. They also introduced tLASCA, a technique in which averaging in the spatial domain is performed on contrast maps obtained using LSI. They showed that tLASCA give better results and is faster than SLASCA and LSI.

All techniques discussed here have advantages and disadvantages. Imaging blood flow using LASCA gives a higher temporal resolution compared to LSI and LSFG, so for fast-changing perfusion levels it is the best candidate. LSI on the other hand provides the best spatial resolution, which makes it suitable for producing detailed perfusion images. LSFG is a combination between these two techniques, which makes it ideal for situations where a trade-off between temporal and spatial resolution is needed.

Usually, a speckle pattern is built up from a dynamic and a static part. As is shown by Yuan et al. [41], the static part does not influence the contrast in LSI, which results in a higher SNR for LSI compared to LASCA and LSFG.

\section{Applications}

The laser speckle contrast techniques discussed above can be used in a wide variety of biomedical applications, and several researchers have presented in-vivo results. DaCosta [57] used it to monitor the heartbeat 
of a human volunteer in a non-invasive way. Sadhwani et al. [58] showed that the thickness of a Teflon layer could be determined by using laser speckle contrast techniques, so both, they and Zimnyakov and Misnin [39], suggested that laser speckle contrast techniques could be used for burn depth diagnosis.

Richards and Briers [36] showed that contrast images obtained using LASCA give a good picture of the movement of red blood cells in the hand of a volunteer. Cheng and Duong [49] and Konishi et al. [54] even used LSI and LSFG, respectively, to map the ocular blood flow in the retina.

Ramirez-San-Juan et al. [23] used chicken chorioallantoic membrane (CAM) to prove that the use of the Gaussian-based approach reveals more details such as small vessels than the Lorentzian-based approach.

Several researchers reported contrast images of perfusion in rodents [5, 41, 42, 45, 55, 56, 59-64]. Yuan et al. [41] used changes in contrast images of the rat brain after electrical stimulation to obtain the optimal exposure time. Kubota [59] used LSFG to investigate the effects of diode laser therapy on blood flow in skin flaps in the rat model. To assess changes in blood flow during photo dynamic therapy (PDT), Kruijt et al. [62] used LSPI to monitor the vasculature response in arteries, veins and tumor microvasculature in a rat skin-fold observation chamber. Smith et al. [63] used contrast images to image the microvascular blood flow using an in vivo rodent dorsal skin-fold model during PDT, pulsed dye laser (PDL) irradiation and a combination of both on port wine stains. Dunn et al. [5] used LASCA to map the cerebral flow of a rat and simultaneously measure the perfusion using a laser Doppler probe. They showed that there is a good agreement between the flow in-vivo measured with both techniques. Several researchers like Cheng et al. [42], Tan et al. [55], Li et al. [45], Murari et al. [65] and Le et al. [56], did similar work to image the cerebral flow but used temporal averaging. Zhu et al. [64] monitored thermal-induced changes in tumor blood flow and microvessels in mice by using LASCA, and showed that deformation of vessel is a main factor for changing the blood perfusion of a microvessel.

Besides visualizing blood flow, LASCA can also be used to characterize the composition of atherosclerotic plaques, as achieved by Nadkarni et al. [66, 67], who measured the speckle decorrelation time $\tau_{c}$, which provides an index of plaque viscoelasticity and helps characterize the composition of the plaque, which can be used to identify high-risk lesions. They showed that LASCA is highly sensitive to changes in the plaque composition so it can be used to identify thin-cap fibroatheromas.

\section{Comparison with laser Doppler perfusion imaging}

Currently, there are two major techniques that are used to image tissue perfusion. Besides laser speckle contrast techniques, laser Doppler perfusion imaging (LDPI) is used to image the perfusion. In LDPI, optical Doppler shifts are analyzed from the temporal intensity fluctuations that are caused by the dynamic speckle pattern. A number of locally measured power spectra of these intensity fluctuations is converted into a perfusion image.

Until recently, LASCA had the advantage over LDPI of being a full-field technique, whereas LDPI was a scanning technique. This scanning mode resulted in long measurement times, which made LDPI less favorable for the clinical environment. This advantage decreased when LDPI became a full-field technique by introducing a high-speed CMOS camera for the detection of the Doppler-shifted light [68-71]. From that moment on, both techniques had a measurement time in the millisecond range.

The introduction of the high-speed CMOS cameras in LDPI directly reveals another advantage of LASCA over LDPI. To perform LASCA measurements, an inexpensive camera that can achieve a frame-rate of $200 \mathrm{~Hz}$ (i.e., an integration time of $5 \mathrm{~ms}$ ) is sufficient, whereas for LDPI, a state-of-the-art high-speed camera that can achieve a frame-rate of about $25 \mathrm{kHz}$ is needed.

On the other hand, the physics behind LDPI is well known and it is shown that, for low blood concentrations, the concentration of red blood cells and their average velocity are both linearly represented by the perfusion estimation given in LDPI. Bonner and Nossal [22] published a widely accepted theoretical model of laser Doppler measurements to determine these parameters of blood flow in tissue.

For LASCA and related speckle contrast techniques, a model linking the measurement outcome to the perfusion, is not available. The reading of LASCA is based on blurring of the speckles on the detector. To link this blurring with the average velocity of red blood cells, assumptions should be made about an appropriate velocity distribution (e.g., Lorentzian, Gaussian, Voigt) the fraction of moving red blood cells and other parameters (e.g., particle size). With the wide variety of biological applications, this is a major challenge. So yet there is no proper model linking the speckle contrast to the perfusion. To our knowledge, determination of the concentration of red blood cells with LASCA has not been shown to be possible.

Another difference between laser speckle contrast techniques and LDPI is the opportunity to apply high- 
Fig. 4 LASCA contrast maps of the heart of a chicken embryo. a taken with an integration time of $15 \mathrm{~ms}$ and b taken with an integration time of $40 \mathrm{~ms}$. The black arrows indicate the heart and the major feeding vessel

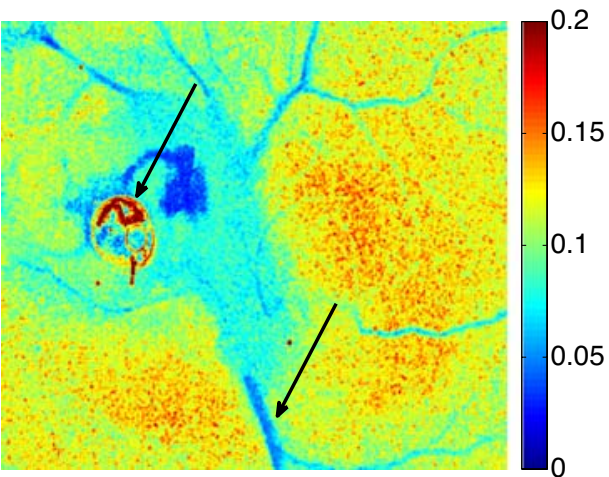

(a)

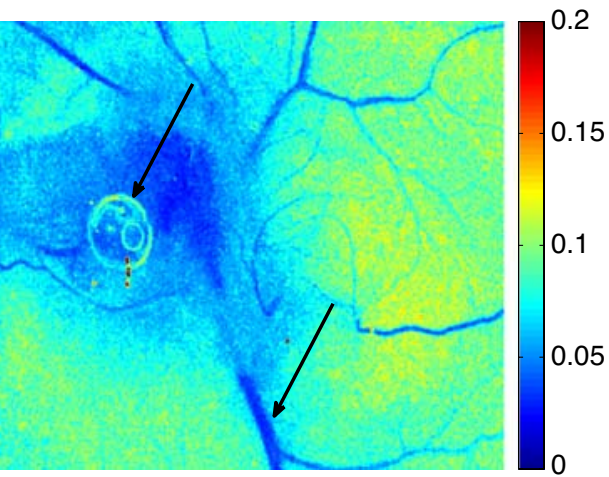

(b) pass filtering (e.g., above $100 \mathrm{~Hz}$ ) to the recorded signals in LDPI to filter out movement artifacts. For laser speckle contrast techniques, filtering out those artifacts is not possible, which is a major disadvantage. Another disadvantage of laser speckle contrast techniques is shown in Fig. 4. The exposure time is a parameter that can be chosen freely, however, the choice of the integration time influences the calculated contrast values drastically. Both contrast images in Fig. 4 show the same area of growing blood vessels of a chicken embryo and its chorio-allantoic membrane. The black arrows in the images indicate the heart and the major feeding vessel. With a short integration time (i.e., $15 \mathrm{~ms}$, Fig. 4a) the contrast image shows mainly fast-moving blood cells (i.e., blood vessels around the heart) whereas with a long integration time (i.e., $40 \mathrm{~ms}$, Fig. 4b) the contrast image highlights slower moving blood cells (i.e., blood vessels further away from the heart).
So the choice of the integration time determines what can be seen in the image.

To illustrate how images look like produced by several contrast techniques discussed here, Fig. 5 shows the same sample imaged with LASCA, LSI, LSFG, and compared with LDPI. With capsicum cream (Midalgan, Remark Groep BV, Meppel, the Netherlands), a perfusion increasing cream, a pattern was written on the right hand of a volunteer (male, $28 \mathrm{yr}$ ). The tissue was imaged twice, once with a frame rate of $125 \mathrm{~Hz}$ (an integration time of $8 \mathrm{~ms}$ ) for LASCA, LSI and LSFG and once with a frame rate of $27 \mathrm{kHz}$ (an integration time of $37 \mu \mathrm{s}$ ) for LDPI. In each measurement, the f-number was chosen to avoid saturation. The data obtained at a low frame rate were processed with LASCA $(5 \times 5$ pixels), LSI (25 images) and LSFG, the resulting images are shown in Fig. 5a-c, respectively. The second measurement was processed with LDPI (i.e., first moment

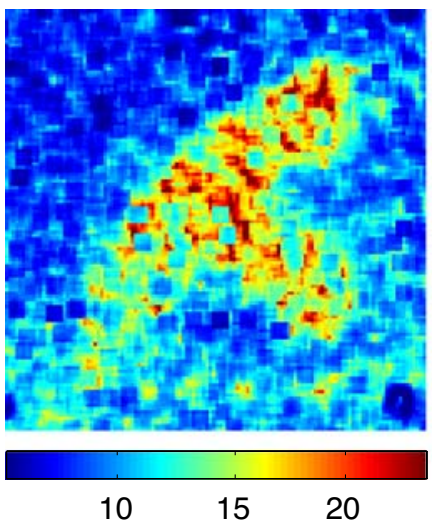

(a)

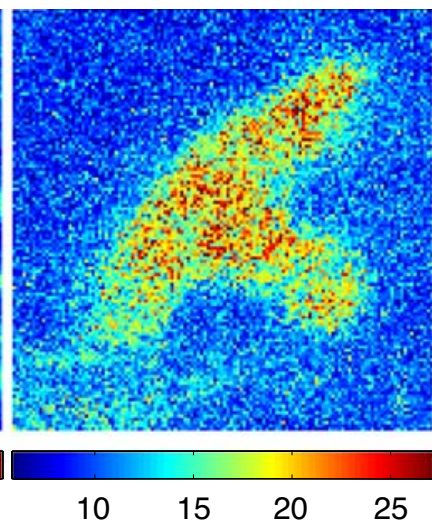

(b)

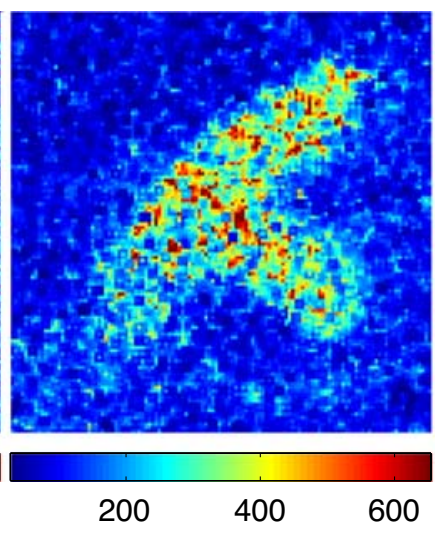

(c)

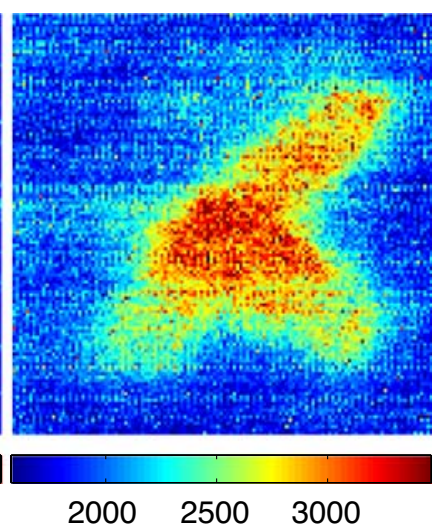

(d)

Fig. 5 Comparison of three speckle contrast techniques discussed here with laser Doppler perfusion imaging. On the right hand of a volunteer a pattern was written with capsicum cream, a perfusion increasing cream, and imaged with the different techniques. a The inverse of the contrast determined with LASCA.

b the inverse of the contrast determined with LSI. c MBR determined with LSFG and d the perfusion determined with LDPI. The contrast, MBR and perfusion values are shown in the colorbar 
of the power spectrum from 0 to $13.5 \mathrm{kHz}$ ) and normalized with DC, the resulting image is shown in Fig. 5d. In comparison with LDPI, LSI and LSFG give a good indication of areas with high and low perfusion. The lack of spatial resolution of LASCA compared to the other techniques is also clearly illustrated.

Forrester et al. [52] compared LDPI with some of the laser speckle contrast techniques discussed in this paper. They imaged digits of a human hand and the joint capsule and muscle in a rabbit knee, and suggested that laser speckle contrast techniques are a good and fast alternative for LDPI and therefore should be further developed. Furthermore, the higher temporal resolution of LASCA made it more sensitive to the hyperaemic response after an occlusion. Besides Forrester et al., several other researchers have performed a comparison between both techniques [51, 71-73]. Briers [51] compared both techniques from a more theoretical point of view and postulated the essential equivalence of both techniques. He therefore encouraged some cross fertilization of ideas between both techniques. Serov and Lasser [71] compared LASCA and LDI in their hybrid imaging system. They not only compared imaging quality and speed but also sensitivity for flow parameters such as speed and concentration. In their measurements, LASCA turned out be faster (i.e., ten frames per second) but had a poorer spatial resolution. Thompson and Andrews [73] postulated a method to gain the quantitative advantages of LDPI while keeping the speed of LASCA. They claim that by making use of a temporal autocorrelation function of the LASCA measurement, a perfusion index comparable to the index of LDPI can be obtained.

\section{Conclusions}

Speckle contrast techniques are gaining interest in the field of tissue perfusion imaging. In this paper, we have presented the principles and various implementations of the speckle contrast methods, reviewed the contribution of these techniques to the field of perfusion imaging and described their technical development.

Speckle contrast techniques have advantages over their main counterpart, laser Doppler perfusion imaging (LDPI). Speckle contrast techniques need only one or a few frames to determine the tissue perfusion, which makes it fast. They also need a low-frame-rate camera only, which makes them inexpensive techniques. However, they also have one major disadvantage with respect to LDPI; the readings in LDPI can be related to the Doppler effect, which is described by a theory that is widely accepted and understood. For LASCA this is not the case, since, for example, it is still unknown which velocity distribution (e.g., Voigt, Lorentzian, or Gaussian) should be used. The need to assume a specific velocity distribution to relate the speckle contrast to the tissue perfusion makes the technique less generally applicable.

Once there is a consensus about a theoretical model for LASCA that connects the contrast unambiguously to the perfusion level, it can become one of the leading techniques for measuring tissue-perfusion maps.

Acknowledgements We thank Jurjen Couperus and Koen Thuijs for their involvement in setting up the LASCAinstrument. This work has been funded by the Technology Foundation STW (Grant No 06443) and Perimed AB, Sweden.

Open Access This article is distributed under the terms of the Creative Commons Attribution Noncommercial License which permits any noncommercial use, distribution, and reproduction in any medium, provided the original author(s) and source are credited.

\section{References}

1. Fuji H, Asakura T, Nohira K, Shintomi Y, Ohura T (1985) Blood flow observed by time-varying laser speckle. Opt Lett 10:104-106

2. Ruth B (1990) Blood flow determination by the laser speckle method. Int J Microcirc Clin Exp 9:21-45

3. Ulyanov SS (1998) Speckled speckle statistics with a small number of scatterers: implication for blood flow measurement. J Biomed Opt 3:237-245

4. Ulyanov SS, Tuchin VV (2000) Use of low-coherence speckled speckles for bioflow measurements. Appl Opt 39:63856389

5. Dunn AK, Bolay H, Moskowitz MA, Boas DA (2001) Dynamic imaging of cerebral blood flow using laser speckle. J Cereb Blood Flow Metab 21:195-201

6. Bray R, Forrester K, Leonard C, McArthur R, Tulip J, Lindsay R (2003) Laser Doppler imaging of burn scars: a comparison of wavelength and scanning methods. Burns 29:199-206

7. Stewart CJ, Frank R, Forrester KR, Tulip J, Lindsay R, Bray RC (2005) A comparison of two laser-based methods for determination of burn scar perfusion: laser Doppler versus laser speckle imaging. Burns 31:744-752

8. La Hei E, Holland A, Martin H (2006) Laser Doppler imaging of paediatric burns: burn wound outcome can be predicted independent of clinical examination. Burns 32:550-553

9. de Mul FFM, Blaauw J, Aarnoudse JG, Smit AJ, Rakhorst G (2007) Diffusion model for iontophoresis measured by laser-Doppler perfusion flowmetry, applied to normal and preeclamptic pregnancies. J Biomed Opt 12:14032-1

10. Leahy MJ, Enfield JG, Clancy NT, O'Doherty J, McNamara $\mathrm{P}$, Nilsson GE (2007) Biophotonic methods in microcirculation imaging. Med Laser Appl 22:105-126

11. Wårdell K, Jakobsson A, Nilsson GE (1993) Laser Doppler perfusion imaging by dynamic light scattering. IEEE Trans Biomed Eng 40:309-316

12. Webster S, Briers JD (1994) Time-integrated speckle for the examination of movement in biological systems. In: 
Cerullo LJ, Heiferman KS, Liu H, Podbielska H, Wist AO, Zamorano LJ (eds) Clinical applications of modern imaging technology II, vol 2132. Proc. SPIE, pp 444-452

13. Zhao H, Webb RH, Ortel B (2002) Review of noninvasive methods for skin blood flow imaging in microcirculation. J Clin Eng 27:40-47

14. Aizu Y, Asakura T (1991) Bio-speckle phenomena and their application to the evaluation of blood flow. Opt Laser Technol 23:205-219

15. Briers JD (2001) Laser Doppler, speckle and related techniques for blood perfusion mapping and imaging. Physiol Meas 22:R35-R66

16. Briers JD (2007) Laser speckle contrast imaging for measuring blood flow. Opt Appl XXXVII:139-152

17. Duncan DD, Kirkpatrick SJ, Wang RK (2008) Statistics of local speckle contrast. J Opt Soc Am A, Opt Image Sci Vis 25:9-15

18. Goodman JW, Parry G (1984) Laser speckle and related phenomena. Springer, Berlin Heidelberg New York

19. Briers JD, Webster S (1995) Quasi real-time digital version of single-exposure speckle photography for full-field monitoring of velocity or flow fields. Opt Commun 116:36-42

20. Briers JD, Richards GJ (1997) Laser speckle contrast analysis (LASCA) for flow measurement. In: Gorecki C (ed) Optical inspection and micromeasurements II. Proc. SPIE, vol 3098, pp 211-221

21. Richards G, Briers J (1997) Laser speckle contrast analysis (LASCA): a technique for measuring capillary blood flow using the first-order statistics of laser speckle patterns. In: IEE colloquium (Digest), vol 124. London, UK, pp 11-1

22. Bonner R, Nossal R (1981) Model for laser Doppler measurements of blood flow in tissue. Appl Opt 20:20972107

23. Ramirez-San-Juan JC, Nelson JS, Choi B (2006) Comparison of Lorentzian and Guassian-based approaches for laser speckle imaging of blood flow dynamics. In: Tuchin VV, Izatt JA, Fujimoto JG (eds) Coherence domain optical methods and optical coherence tomography in biomedicine X. Proc. SPIE, vol 6079, pp 380-383

24. Fercher AF, Briers JD (1981) Flow visualization by means of single-exposure speckle photography. Opt Commun 37:326330

25. Ramirez-San-Juan JC, Ramos-Garcia R, Guizar-Iturbide I, Martinez-Niconoff G, Choi B (2008) Impact of velocity distribution assumption on simplified laser speckle imaging equation. Opt Express 16:3197-3203

26. Duncan DD, Kirkpatrick SJ, Gladish JC (2008) What is the proper statistical model for laser speckle flowmetry? In: Tuchin VV, Wang LV (eds) Complex dynamics and fluctuations in biomedical photonics V. Proc. SPIE, vol 6855, pp 685502-685502-7

27. Archbold E, Ennos AE (1972) Displacement measurement from double-exposure laser photographs. Opt Acta 19:253271

28. Grousson R, Mallick S (1977) Study of flow pattern in a fluid by scattered laser light. Appl Opt 16:2334-2336

29. Iwata K, Hakoshima T, Nagata R (1978) Measurement of flow velocity distribution by multiple-exposure speckle photography. Opt Commun 25:311-314

30. Iwai T, Shigeta K (1990) Experimental study on the spatial correlation properties of speckled speckles using digital speckle photography. Jpn J Appl Phys 29:1099-1102

31. Ganilova Y, Li P, Zhu D, Lin N, Chen H, Luo Q, Ulyanov S (2006) Digital speckle-photography, LASCA and crosscorrelation techniques for study of blood microflow in isolated vessel. In: Tuchin VV (ed) Saratov fall meeting 2005: optical technologies in biophysics and medicine VII. Proc. SPIE, vol 6163. SPIE, 616319

32. Briers JD, Webster S (1996) Laser speckle contrast analysis LASCA): a nonscanning, full-field technique for monitoring capillary blood flow. J Biomed Opt 1:174-179

33. Briers JD (2001) Time-varying laser speckle for measuring motion and flow. In: Zimnyakov DA (ed) Coherent optics of ordered and random media, vol 4242, pp 25-39

34. He XW, Briers JD (1998) Laser speckle contrast analysis (LASCA): a real-time solution for monitoring capillary blood flow and velocity. In: Hoffman EA (ed) Physiology and function from multidimensional images. Proc. SPIE, vol 3337, pp 98-107

35. Briers JD, Richards G, He XW (1999) Capillary blood flow monitoring using laser speckle contrast analysis (LASCA). J Biomed Opt 4:164-175

36. Richards GJ, Briers JD (1997) Capillary-blood-flow monitoring using laser speckle contrast analysis (LASCA): improving the dynamic range. In: Tuchin VV, Podbielska H, Ovryn B (eds) Coherence domain optical methods in biomedical science and clinical applications. Proc. SPIE, vol 2981, pp 160 171

37. Briers JD, He XW (1998) Laser speckle contrast analysis (LASCA) for blood flow visualization: improved image processing. In: Priezzhev AV, Asakura T, Briers JD (eds) Optical diagnostics of biological fluids III. Proc. SPIE, vol 3252, pp 26-33

38. Zimnyakov DA, Mishin AB, Bednov AA, Cheung C, Tuchin VV, Yodh AG (1999) Time-dependent speckle contrast measurements for blood microcirculation monitoring. In: Priezzhev AV, Asakura T (eds) Optical diagnostics of biological fluids IV. Proc. SPIE, vol 3599, pp 157-166

39. Zimnyakov DA, Misnin AB (2001) Blood microcirculation monitoring by use of spatial filtering of time-integrated speckle patterns: potentialities to improve the depth resolution. In: Priezzhev AV, Cote GL (eds) Optical diagnostics and sensing of biological fluids and glucose and cholesterol monitoring. Proc. SPIE, vol 4263, pp 73-82

40. MacKintosh FC, Zhu JX, Pine DJ, Weitz DA (1989) Polarization memory of multiply scattered light. Phys Rev B 40:93429345

41. Yuan S, Devor A, Boas DA, Dunn AK (2005) Determination of optimal exposure time for imaging of blood flow changes with laser speckle contrast imaging. Appl Opt 44:1823-1830

42. Cheng H, Luo Q, Zeng S, Chen S, Cen J, Gong H (2003) Modified laser speckle imaging method with improved spatial resolution. J Biomed Opt 8:559-564

43. Choi B, Ramirez-San-Juan JC, Lotfi J, Nelson JS (2006) Linear response range characterization and in vivo application of laser speckle imaging of blood flow dynamics. J Biomed Opt 11:041129

44. Nothdurft R, Yao G (2005) Imaging obscured subsurface inhomogeneity using laser speckle. Opt Express 13:1003410039

45. Li P, Ni S, Zhang L, Zeng S, Luo Q (2006) Imaging cerebral blood flow trough the intact rat skull with temporal laser speckle imaging. Opt Lett 31:1824-1826

46. Völker AC, Zakharov P, Weber B, Buck F, Scheffold F (2005) Laser speckle imaging with an active noise reduction scheme. Opt Express 13:9782-9787

47. Bandyopadhyay R, Gittings AS, Suh SS, Dixon PK, Durian DJ (2005) Speckle-visibility spectroscopy: a tool to study time-varying dynamics. Rev Sci Instrum 76:093110

48. Zakharov P, Völker A, Buck A, Weber B, Scheffold F (2006) Quantitative modeling of laser speckle imaging. Opt Lett 31:3465-3467 
49. Cheng H, Duong TQ (2007) Simplified laser-speckle-imaging analysis method and its application to retinal blood flow imaging. Opt Lett 32:2188-2190

50. Parthasarathy AB, Tom WJ, Gopal A, Zhang X, Dunn AK (2008) Robust flow measurement with multi-exposure speckle imaging. Opt Express 16:1975-1989

51. Briers JD (1996) Laser Doppler and time-varying speckle: a reconciliation. J Opt Soc Am A 13:45-350

52. Forrester KR, Stewart C, Tulip J, Leonard C, Bray RC (2002) Comparison of laser speckle and laser Doppler perfusion imaging: measurement in human skin and rabbit articular tissue. Med Biol Eng Comput 40:687-697

53. Forrester KR, Tulip J, Leonard C, Bray RC, Robert C (2004) A laser speckle imaging technique for measuring tissue perfusion. IEEE Trans Biomed Eng 51:2074-2084

54. Konishi N, Tokimoto Y, Kohra K, Fujii H (2002) New laser speckle flowgraphy system using CCD camera. Opt Rev 9:163-196

55. Tan YK, Liu WZ, Yew YS, Ong SH, Paul JS (2004) Speckle image analysis of cortical blood flow and perfusion using temporally derived contrasts. In: International conference on image processing ICIP 2004. Proc. IEEE, vol 5, pp 3323-3326

56. Le TM, Paul JS, Al-Nashash H, Tan A, Luft AR, Sheu FS, Ong SH (2007) New insights into image processing of cortical blood flow monitors using laser speckle imaging. IEEE Trans Biomed Eng 26:833-842

57. DaCosta G (1995) Optical remote sensing of heartbeats. Opt Commun 117:395-398

58. Sadhwani A, Schomacker KT, Tearney GJ, Nishioka NS (1996) Determination of teflon thickness with laser speckle. i. potential for burn depth diagnosis. Appl Opt 35:57275735

59. Kubota J (2002) Effects of diode laser therapy on blood flow in axial pattern flaps in the rat model. Lasers Med Sci 17:146153

60. Choi B, Kang NM, Nelson JS (2004) Laser speckle imaging for monitoring blood flow dynamics in the in vivo rodent dorsal skin fold model. Microvasc Res 68:143-146

61. Paul JS, Luft AR, Yew E, Sheu FS (2006) Imaging the development of an ischemic core following photochemically induced cortical infarction in rats using laser speckle contrast analysis (LASCA). Neuroimage 29:38-45

62. Kruijt B, de Bruijn HS, van der Ploeg-van den Heuvel A, Sterenborg HJCM, Robinson DJ (2006) Laser speckle imaging of dynamic changes in flow during photodynamic therapy. Lasers Med Sci 21:208-212

63. Smith TK, Choi B, Ramirez-San-Juan JC, Nelson JS, Osann K, Kelly KM (2006) Microvascular blood flow dynamics associated with photodynamic therapy, pulsed dye laser irradiation and combined regimens. Lasers Surg Med 38:532-539

64. Zhu D, Lu W, Weng Y, Cui H, Luo Q (2007) Monitoring thermal-induced changes in tumor blood flow and microvessels with laser speckle contrast imaging. Appl Opt 46:19111917

65. Murari K, Li N, Rege A, Jia X, All A, Thakor N (2007) Contrast-enhanced imaging of cerebral vasculature with laser speckle. Appl Opt 46:5340-5346

66. Nadkarni SK, Bouma BE, Helg T, Chan R, Halpern E, Chau A, Minsky MS, Motz JT, Houser SL, Tearney GJ (2005) Characterization of atheroslerotic plaques by laser speckle imaging. Circulation 112:885-892

67. Nadkarni SK, Bouma BE, de Boer J, Tearney GJ (2008) Evaluation of collagen in atherosclerotic plaques: the use of two coherent laser-based imaging methods. Lasers Med Sci doi:10.1007/s10103-007-0535-x

68. Serov A, Steenbergen W, de Mul FFM (2002) Laser Doppler perfusion imaging with a complimentary metal oxide semiconductor image sensor. Opt Lett 27:300-302

69. Serov A, Steinacher B, Lasser T (2005) Full-field laser Doppler perfusion imaging and monitoring with an intelligent CMOS camera. Opt Express 13:3681-3689

70. Serov A, Lasser T (2005) High-speed laser Doppler perfusion imaging using an integrating CMOS image sensor. Opt Express 13:6416-6428

71. Serov A, Lasser T (2006) Combined laser Doppler and laser speckle imaging for real-time blood flow measurements. In: Coté GL, Priezzhev AV (eds) Optical diagnostics and sensing VI. Proc. SPIE, vol 6094, pp 33-40

72. Draijer MJ, Hondebrink E, van Leeuwen TG, Steenbergen W (2008) Connecting laser Doppler perfusion imaging and laser speckle contrast analysis. In: Coté GL, Priezzhev AV (eds) Optical diagnostics and sensing VIII. Proc. SPIE, vol 6863, pp 68630C-68630C-8

73. Thompson OB, Andrews MK (2008) Spectral density and tissue perfusion from speckle contrast measurements. In: Izatt JA, Fujimoto JG, Tuchin VV (eds) Coherence domain optical methods and optical coherence tomography in biomedicine XII. Proc. SPIE, vol 6847, pp 68472D-68472D-7 